\title{
Stability of Stochastic Reaction-Diffusion Systems with Markovian Switching and Impulsive Perturbations
}

\author{
Yanbo $\mathrm{Li}^{1,2}$ and Yonggui $\mathrm{Kao}^{1}$ \\ ${ }^{1}$ Department of Mathematics, Harbin Institute of Technology, Weihai 264209, China \\ ${ }^{2}$ School of Mathematical Sciences, Guangxi Teachers Education University, Guangxi 530001, China
}

Correspondence should be addressed to Yonggui Kao, kaoyonggui@sina.com

Received 4 September 2012; Accepted 23 September 2012

Academic Editor: Alexei Mailybaev

Copyright (C) 2012 Y. Li and Y. Kao. This is an open access article distributed under the Creative Commons Attribution License, which permits unrestricted use, distribution, and reproduction in any medium, provided the original work is properly cited.

This paper is devoted to investigating mean square stability of a class of stochastic reactiondiffusion systems with Markovian switching and impulsive perturbations. Based on Lyapunov functions and stochastic analysis method, some new criteria are established. Moreover, a class of semilinear stochastic impulsive reaction-diffusion differential equations with Markovian switching is discussed and a numerical example is presented to show the effectiveness of the obtained results.

\section{Introduction}

Markovian jump systems, introduced by Krasovskiř and Lidskiř [1] in 1961, have received increasing attention, see [2-15] and references therein. Shi and Boukas [3] have probed $H_{\infty}$ control for Markovian jumping linear systems with parametric uncertainty. Zhang et al. [4-6] have discussed markovian jump linear systems with partly unknown transition probability. Mao et al. [7-13] have established a number of stability criteria for stochastic differential equations with Markovian switching. However, impulsive perturbations have not been included in the above results.

In fact, impulsive effects widely exist in many fields, such as medicine and biology, economics, mechanics, electronics, and telecommunications [16-19]. Recently, impulsive stochastic differential equations have attracted more and more researchers [20-27]. L. Xu and $\mathrm{D} . \mathrm{Xu}[20]$ have investigated mean square exponential stability of impulsive control stochastic systems with time-varying delay. Li [23] has obtained the attracting set for impulsive stochastic difference equations with continuous time. Pan and Cao [24] have considered 
exponential stability of impulsive stochastic functional differential equations. Zhang et al. [25] have studied stability of impulsive stochastic differential equations in terms of two measures via perturbing Lyapunov functions. Moreover, Markovian Jump Systems with impulsive perturbations have been investigated [28-31]. Zhang et al. [28] have established several criteria for stochastic stability analysis of neutral-type impulsive neural networks with mixed time-varying delays and Markovian jumping. Zhu and Cao [30] have obtained several sufficient conditions on stability of Markovian jump neural networks with impulse control and time-varying delays.

Besides impulsive and stochastic effects, reaction diffusion phenomena cannot be ignored in real systems [32-42]. Kao et al. [34] have discussed exponential stability of impulsive stochastic fuzzy reaction-diffusion Cohen-Grossberg neural networks with mixed delays. Wang et al. [40] have probed stochastic exponential stability of the delayed reactiondiffusion recurrent neural networks with Markovian jumping parameters. However, to the best of our knowledge, there are few considering the Markovian jump systems with impulsive perturbations and reaction-diffusion effects.

Motivated by the above discussions, in this paper, we consider mean square stability of a class of impulsive stochastic reaction-diffusion differential systems with Markovian switching. In Section 2, model description and preliminaries are presented. In Section 3, by utilizing Lyapunov function and stochastic analysis, we obtain some new conditions ensuring mean square stability of impulsive stochastic reaction-diffusion differential equations with Markovian switching. Moreover, mean square stability of a class of semilinear stochastic impulsive reaction-diffusion systems has also been discussed. In Section 4, an example is provided. Section 5 is conclusions.

\section{Model Description and Preliminaries}

In this section, we investigate the impulsive stochastic reaction diffusion equations with Markovian switching described by

$$
\begin{aligned}
d u(t, x)= & {[D(t, x, u) \Delta u+f(t, x, u, \gamma(t))] d t } \\
& +\sigma(t, x, u, \gamma(t)) d w(t), \quad t \geq 0, t \neq t_{k}, x \in G, \\
u\left(t_{k}, x\right)= & H_{k}\left(u\left(t_{k}^{-}, x\right), \gamma\left(t_{k}\right)\right), \quad x \in G,
\end{aligned}
$$

with boundary condition

$$
\left.\frac{\partial u}{\partial N}\right|_{\partial G}=0, \quad t \geq 0
$$

and initial condition

$$
u\left(0, x, i_{0}\right)=\varphi(x), \quad x \in G, i_{0} \in \mathbb{S}
$$

where $u(t, x)=\left(u_{1}(t, x), \ldots, u_{n}(t, x)\right)^{\top}, x=\left(x_{1}, \ldots, x_{m}\right)^{\top} \in G \subset R^{m}, G$ is a bounded set with smooth boundary $\partial G, \partial / \partial N$ is the outward normal derivative. $t_{k}$ is the impulsive 
moment satisfying $0=t_{0}<t_{1}<t_{2}<\cdots$, and $\lim _{k \rightarrow \infty} t_{k}=+\infty, u\left(t_{k}^{+}, x\right)$ and $u\left(t_{k^{\prime}}^{-}, x\right)$ denote the right-hand limit and left-hand limit of $u(t, x)$ at $t_{k}$, respectively. $u(t, x) \in P C\left[[0,+\infty) \times G, R^{n}\right]=\left\{u(t, x):[0,+\infty) \times G \rightarrow R^{n} \mid u(t, x)\right.$ is continuous for all $t \geq 0$ but points $t_{k}, u\left(t_{k^{+}}^{+} x\right)$ and $u\left(t_{k^{\prime}}^{-}, x\right)$ exist, furthermore, $u\left(t_{k^{+}}^{+} x\right)=$ $u\left(t_{k}, x\right), k=\{1,2, \ldots\} . D(t, x, u)$ is a $n \times n$ matrix, $\Delta u=\left\{\Delta u_{1}, \ldots, \Delta u_{n}\right\}^{\top}, \Delta u_{i}=$ $\sum_{l=1}^{m}\left(\partial^{2} u_{i} / \partial x_{i}^{2}\right), i=1,2, \ldots, n$. $f$ and $\sigma$ are continuous, in addition, $f(t, x, u, \gamma(t))=$ $\left\{f_{1}(t, x, u, \gamma(t)), \ldots, f_{n}(t, x, u, \gamma(t))\right\}^{\top}, \sigma(t, x, u, \gamma(t))=\left\{\sigma_{1}(t, x, u, \gamma(t)), \ldots, \sigma_{n}(t, x, u, \gamma(t))\right\}^{\top}$. $H_{k}\left(u\left(t_{k}^{-}, x\right), \gamma(t)\right)=\left\{H_{1 k}\left(u\left(t_{k^{-}}, x\right), \gamma(t)\right), \ldots, H_{n k}\left(u\left(t_{k^{-}}, x\right), \gamma(t)\right)\right\}^{\top}$ represents the impulsive perturbation of $u$ at time $t_{k}$. $w(t)$ is a one-dimensional standard Brownian motion on a complete probability space $\left(\Omega, F,\left\{F_{t}\right\}_{t \geq 0}, P\right)$ with a natural filtration $\left\{F_{t}\right\}_{t \geq 0} .\{\gamma(t), t \geq 0\}$ is a left-continuous Markov process on the probability space $\left(\Omega, F,\left(F_{t}\right)_{t \in I}, P\right)$ and takes values in the finite space $\mathbb{S}=\{1,2, \ldots, \widetilde{N}\}$ with generator $\Lambda=\left(\pi_{i j}\right)(i, j \in \mathbb{S})$ given by

$$
P\{\gamma(t+\Delta)=j \mid \gamma(t)=i\}= \begin{cases}\pi_{i j} \Delta+o(\Delta), & \text { if } i \neq j, \\ 1+\pi_{i i} \Delta+o(\Delta), & \text { if } i=j,\end{cases}
$$

where $\Delta>0$ and $\lim _{\delta \rightarrow 0} O(\Delta) / \Delta=0, \pi_{i j} \geq 0$ is the transition rate from $i$ to $j$ if $i \neq j$ and $\pi_{i i}=-\sum_{j \neq i} \pi_{i j}$. We suppose that the Markov chain $\gamma(\cdot)$ is independent of the Brownian motion $W(\cdot)$. Moreover, we assume that $H_{k}\left(0, \gamma_{0}\right)=f\left(t, x, 0, \gamma_{0}\right)=\sigma\left(t, x, 0, \gamma_{0}\right)=0$, then system (2.1) admits a trivial solution $u=0$. For $u(t, x)=\left(u_{1}(t, x), \ldots, u_{n}(t, x)\right)^{\top} \in R^{n}$, we define $\|u(t, x)\|_{G}=\left(\int_{G}|u(t, x)|^{2} d x\right)^{1 / 2}$ where $|u(t, x)|^{2}=\sum_{i=1}^{n} u_{i}^{2}(t, x)$. For simplicity, we denote $\|u(t, x)\|_{G}$ by $\bar{u}(t)$ throughout this paper.

Let $u\left(t, x ; 0, \varphi, i_{0}\right)$ stand for the solution of system (2.1)-(2.4) through $\left(0, \varphi, i_{0}\right)$.

Definition 2.1. The trivial solution $u=0$ is said to be mean square stable if for any $\varepsilon>0$, there exists $\delta=\delta(\varepsilon)$ such that for all $i_{0} \in \mathbb{S}$, we have

$$
E\left\{\left\|u\left(t, x ; 0, \varphi, i_{0}\right)\right\|_{G}^{2}\right\}<\varepsilon, \quad t \geq 0
$$

when $\varphi$ satisfies $E\left\{\|\varphi\|_{G}^{2}\right\} \leq \delta$.

Definition 2.2. The function $V(t, y, \gamma(t)):[0,+\infty) \times R^{+} \times \mathbb{S} \rightarrow R^{+}$belongs to class $v_{0}^{1,2}$ if

(1) for $k=1,2, \ldots$, the function $V$ is once continuously differentiable in $t$ and twice in $y$ on $\left(t_{k-1}, t_{k}\right) \times R^{+} \times \mathbb{S}$, and, in addition, $V\left(t, 0, \gamma_{0}\right)=0$ holds for $t \geq 0$;

(2) $V(t, y, \gamma(t))$ is locally Lipschitzian in $y$;

(3) for each $k=1,2, \ldots$, there exist finite limits

$$
\begin{aligned}
& V(t, q, \gamma(t)) \longrightarrow V\left(t_{k^{-}}^{-}, y, \gamma\left(t_{k}\right)\right), \quad \text { if }(t, q, \gamma(t)) \longrightarrow\left(t_{k}^{-}, y, \gamma\left(t_{k}\right)\right), \\
& V(t, q, \gamma(t)) \longrightarrow V\left(t_{k}^{+}, y, \gamma\left(t_{k}\right)\right), \quad \text { if }(t, q, \gamma(t)) \longrightarrow\left(t_{k}^{+}, y, \gamma\left(t_{k}\right)\right) .
\end{aligned}
$$




\section{Main Results}

In this section, we will discuss mean square stability of the trivial solution of system (2.1)-(2.4). Assume $H_{k}\left(u\left(t_{k^{\prime}}^{-} x\right), \gamma\left(t_{k}\right)\right)$ satisfies $\left\|H_{k}\left(u\left(t_{k^{\prime}}^{-}, x\right), \gamma\left(t_{k}\right)\right)\right\|_{G}^{2} \leq \Gamma_{k}^{2}\left\|u\left(t_{k^{\prime}}^{-} x\right)\right\|_{G^{\prime}}^{2} \Gamma_{k} \geq$ $0, \gamma(t)=i \in \mathbb{S}, k=1,2, \ldots$

Theorem 3.1. If there exist constants $\alpha>0, \beta>0, \kappa>0$ and a Lyapunov function $V(t, \bar{u}(t), i)$ such that for $\gamma(t)=i, i \in \mathbb{S}$, we have the following.

(A1) $\alpha \bar{u}(t) \leq V(t, \bar{u}(t), i) \leq \kappa \bar{u}(t)$.

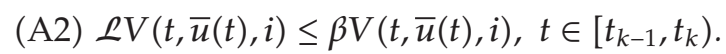

Here the operator $\mathcal{\perp}(t, \bar{u}(t), i)$ is defined as

$$
\begin{aligned}
\mathcal{L}(t, \bar{u}(t), i)= & \frac{\partial V}{\partial t}+\frac{\partial V}{\partial \bar{u}}\left[\int_{G} 2 u^{\top} D(t, x, u) \Delta u d x+\int_{G} 2 u^{\top} f(t, x, u, i) d x\right] \\
& +2 \operatorname{trace}\left\{\left(\int_{G} u^{\top} œ(\mathrm{t}, \mathrm{x}, \mathrm{u}, \mathrm{i}) \mathrm{d} \mathrm{x}\right) \frac{\partial^{2} V}{\partial \overline{\mathrm{u}}^{2}}\left(\int_{\mathrm{G}} \mathrm{u}^{\top} œ(\mathrm{t}, \mathrm{x}, \mathrm{u}, \mathrm{i}) \mathrm{d} \mathrm{x}\right)\right\} \\
& +\sum_{j=1}^{\widetilde{N}} \pi_{i j} V(t, x, j) .
\end{aligned}
$$

(A3) $\lambda>1$, where $\lambda=\inf \left\{\lambda_{k} \mid \lambda_{k}=\alpha / \kappa \gamma^{2}, k=1,2, \ldots\right\}$.

(A4) $\beta\left(t_{k}-t_{k-1}\right)<\ln \lambda, k=1,2, \ldots$

Then, the trivial solution $u=0$ of system (2.1)-(2.4) is stable in mean square.

Proof. For any $\varepsilon>0$, there must exist a scalar $\delta=\delta(\varepsilon)>0$ such that $\delta<(\alpha / \kappa \lambda) \varepsilon$. Next we will prove that $E\left\{\left\|u\left(t, x ; 0, \varphi, i_{0}\right)\right\|_{G}^{2}\right\}<\varepsilon$ if $\varphi$ satisfies $E\left\{\|\varphi\|_{G}^{2}\right\} \leq \delta$.

Let $u=u\left(t, x ; 0, \varphi, i_{0}\right)$. Multiplying both sides of $(2.1)$ by $u^{\top}$, we obtain

$$
\frac{1}{2} d|u|^{2}=\left[u^{\top} D(t, x, u) \Delta u+u^{\top} f(t, x, u, \gamma(t))\right] d t+u^{\top} \sigma(t, x, u, \gamma(t)) d w(t)
$$

By integrating the above equality with respect to $x$ on $G$, we then have

$$
\begin{aligned}
\frac{1}{2} d \int_{G}|u|^{2} d x= & {\left[\int_{G} u^{\top} D(t, x, u) \Delta u d x+\int_{G} u^{\top} f(t, x, u, \gamma(t)) d x\right] d t } \\
& +\int_{G} u^{\top} \sigma(t, x, u, \gamma(t)) d x d w(t) .
\end{aligned}
$$

Namely,

$$
\begin{aligned}
d \bar{u}(t)= & {\left[\int_{G} 2 u^{\top} D(t, x, u) \Delta u d x+\int_{G} u^{\top} f(t, x, u, \gamma(t)) d x\right] d t } \\
& +\int_{G} 2 u^{\top} \sigma(t, x, u, \gamma(t)) d x d w(t)
\end{aligned}
$$


Applying Itô formula, we further compute, when $t \neq t_{k}$,

$$
d V(t, \bar{u}(t), i)=\mathcal{L} V(t, \bar{u}(t), i)+\frac{\partial V}{\partial \bar{u}} \int_{G} 2 u^{\top} \sigma(t, x, u, i) d x d w(t),
$$

where $\gamma(t)=i$ and

$$
\begin{aligned}
& \mathcal{L V}(t, \bar{u}(t), i) \\
& =\frac{\partial V(t, \bar{u}(t), i)}{\partial t}+\frac{\partial V(t, \bar{u}(t), i)}{\partial \bar{u}}\left[\int_{G} 2 u^{\top} D(t, x, u) \Delta u d x+\int_{G} 2 u^{\top} f(t, x, u, i) d x\right] \\
& +2 \operatorname{trace}\left\{\left(\int_{G} u^{\top} \sigma(t, x, u, i) d x\right) \frac{\partial^{2} V}{\partial \bar{u}^{2}}\left(\int_{G} u^{\top} \sigma(t, x, u, i) d x\right)\right\} \\
& +\sum_{j=1}^{\widetilde{N}} \pi_{i j} V(t, x, j)
\end{aligned}
$$

For $t \in\left[t_{k}, t_{k+1}\right)$, integrating (3.5) with respect to $t$ from $t_{k}$ to $t$, one has

$$
\begin{aligned}
V(t, \bar{u}(t), \gamma(t))= & V\left(t_{k}, \bar{u}\left(t_{k}\right), \gamma\left(t_{k}\right)\right)+\int_{t_{k}}^{t} \rho V(s, \bar{u}(s), \gamma(s)) d s \\
& +\int_{t_{k}}^{t} \frac{\partial V}{\partial \bar{u}} \int_{G} 2 u^{\top} \sigma(t, x, u, \gamma(t) d x d w(t)) .
\end{aligned}
$$

Taking the mathematical expectation of both sides of (3.7), we obtain

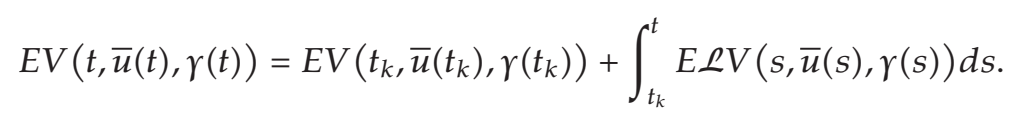

Choosing small enough $\Delta t>0$ such that $t+\Delta t \in\left[t_{k}, t_{k+1}\right)$, it is easy to see that

$$
\begin{aligned}
& E V(t+\Delta t, \bar{u}(t+\Delta t), \gamma(t+\Delta t))=E V\left(t_{k}, \bar{u}\left(t_{k}\right), \gamma\left(t_{k}\right)\right)
\end{aligned}
$$

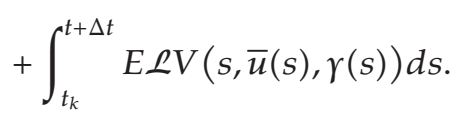

We thus derive from (3.8) and (3.9) that

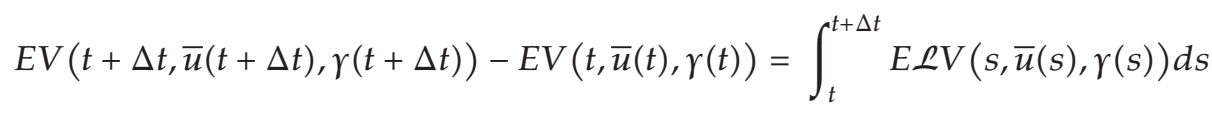

$$
\begin{aligned}
& \leq \int_{t}^{t+\Delta t} \beta E V(s, \bar{u}(s), \gamma(s)) d s .
\end{aligned}
$$


So

$$
D^{+} E V(t, \bar{u}(t), \gamma(t)) \leq \beta E V(t, \bar{u}(t), \gamma(t))
$$

Next, we will first prove

$$
E V(t, \bar{u}(t), \gamma(t)) \leq \kappa \lambda \delta, \quad 0 \leq t<t_{1}
$$

Obviously,

$$
E V\left(0, \bar{u}(0), \gamma_{0}\right) \leq E \kappa \bar{u}(0)=\kappa E\|\varphi\|_{G}^{2} \leq \kappa \delta<\kappa \lambda \delta
$$

If inequality (3.12) does not hold, there must exist some $s \in\left(0, t_{1}\right)$ such that

$$
E V(s, \bar{u}(s), \gamma(s))>\kappa \lambda \delta>\kappa \delta \geq E V\left(0, \bar{u}(0), \gamma_{0}\right) .
$$

Let $s_{1}=\inf \left\{s \in\left(0, t_{1}\right) \mid E V(s, \bar{u}(s), \gamma(s)>\kappa \lambda \delta\}\right.$. Since $E V(t, \bar{u}(t), \gamma(t))$ is continuous on [0, $\left.s_{1}\right]$, there exist $\widehat{s} \in\left(0, s_{1}\right)$ such that

$$
\begin{gathered}
E V(\widehat{s}, \bar{u}(\widehat{s}), \gamma(\widehat{s}))=\kappa \lambda \delta, \\
E V(t, \bar{u}(t), \gamma(t)) \leq \kappa \lambda \delta, \quad t \in[0, \widehat{s}), \\
D^{+} E V(\widehat{s}, \bar{u}(\widehat{s}), \gamma(\widehat{s}))>0 .
\end{gathered}
$$

From $E V(\widehat{s}, \bar{u}(\widehat{s}), \gamma(\widehat{s}))=\kappa \lambda \delta>\kappa \delta$, if $E V\left(0, \bar{u}(0), \gamma_{0}\right) \leq \kappa \delta$, we know that there is $s_{2} \in[0, \widehat{s})$ such that

$$
\begin{gathered}
E V\left(s_{2}, \bar{u}\left(s_{2}\right), \gamma\left(s_{2}\right)\right)=\kappa \delta, \\
E V(t, \bar{u}(t), \gamma(t)) \geq \kappa \delta, \quad t \in\left[s_{2}, \widehat{s}\right], \\
D^{+} E V\left(s_{2}, \bar{u}\left(s_{2}\right), \gamma\left(s_{2}\right)\right)>0 .
\end{gathered}
$$

On the other hand, noticing $D^{+} E V(t, \bar{u}(t), \gamma(t)) \leq \beta E V(t, \bar{u}(t), \gamma(t))$, we obtain

$$
\frac{D^{+} E V(t, \bar{u}(t), \gamma(t))}{E V(t, \bar{u}(t), \gamma(t))} \leq \beta
$$

Integrating both sides of (3.17) on $t \in\left[s_{2}, \widehat{s}\right]$ gives

$$
\int_{s_{2}}^{\widehat{s}} \frac{D^{+} E V(s, \bar{u}(s), \gamma(s))}{E V(s, \bar{u}(s), \gamma(s))} d s \leq \int_{s_{2}}^{\widehat{s}} \beta d s<\beta t_{1}<\ln \lambda
$$


However,

$$
\int_{s_{2}}^{\widehat{s}} \frac{D^{+} E V(s, \bar{u}(s), \gamma(s))}{E V(s, \bar{u}(s), \gamma(s))} d s=\int_{E V\left(s_{2}, \bar{u}\left(s_{2}\right), \gamma\left(s_{2}\right)\right)}^{E V(\widehat{s}, \bar{u}(\widehat{s}), \gamma(\hat{s}))} \frac{d \eta}{\eta}=\int_{\kappa \delta}^{\kappa \lambda \delta} \frac{d \eta}{\eta}=\ln (\kappa \lambda \delta)-\ln (\kappa \delta)=\ln \lambda,
$$

which is a contradiction. Therefore,

$$
E V(t, \bar{u}(t), \gamma(t)) \leq \kappa \lambda \delta, \quad 0 \leq t<t_{1}
$$

Furthermore,

$$
\begin{aligned}
E V\left(t_{1}, \bar{u}\left(t_{1}\right), \gamma\left(t_{1}\right)\right) & \leq E \kappa \bar{u}\left(t_{1}\right)=\kappa E\left\|H_{1}\left(u\left(t_{1}^{-}, x\right)\right)\right\|_{G}^{2} \leq \kappa \Gamma_{1}^{2} E\left\|u\left(t_{1}^{-}, x\right)\right\|_{\Omega}^{2} \\
& \leq \frac{\kappa \Gamma_{1}^{2}}{\alpha} E V\left(t_{1}, \bar{u}\left(t_{1}^{-}, \gamma\left(t_{1}\right)\right)\right) \leq \frac{\kappa \lambda \delta}{\lambda_{1}} \leq \kappa \delta
\end{aligned}
$$

Now we assume that

$$
\begin{gathered}
E V(t, \bar{u}(t), \gamma(t)) \leq \kappa \lambda \delta, \quad t_{m-1} \leq t<t_{m} \\
E V\left(t_{m}, \bar{u}\left(t_{m}\right), \gamma\left(t_{m}\right), \gamma\left(t_{m}\right)\right) \leq \kappa \delta
\end{gathered}
$$

and then prove

$$
\begin{gathered}
E V(t, \bar{u}(t), \gamma(t)) \leq \kappa \lambda \delta, \quad t_{m} \leq t<t_{m+1} \\
E V\left(t_{m}+1, \bar{u}\left(t_{m}+1\right), \gamma\left(t_{m}+1\right)\right) \leq \kappa \delta
\end{gathered}
$$

If not, there must exist some $\tau \in\left(t_{m}, t_{m+1}\right)$ such that

$$
E V(\tau, \bar{u}(\tau), \gamma(\tau))>\kappa \lambda \delta>\kappa \delta \geq E V\left(t_{m}, \bar{u}\left(t_{m}\right), \gamma\left(t_{m}\right), \gamma\left(t_{m}\right)\right)
$$

Let

$$
\tau_{1}=\inf \left\{\tau \in\left(t_{m}, t_{m+1}\right) \mid E V(\tau, \bar{u}(\tau), \gamma(\tau))>\kappa \lambda \delta\right\}
$$

Since $E V(t, \bar{u}(t), \gamma(t))$ is continuous in $\left[t_{m}, \tau_{1}\right]$, there exists $\tau_{2} \in\left(t_{m}, \tau_{1}\right)$ satisfying

$$
\begin{gathered}
E V\left(\tau_{2}, \bar{u}\left(\tau_{2}\right), \gamma\left(\tau_{2}\right)\right)=\kappa \lambda \delta \\
E V(t, \bar{u}(t), \gamma(t)) \leq \kappa \curlywedge \delta, \quad t \in\left[t_{m}, \tau_{2}\right] \\
D^{+} E V\left(\tau_{2}, \bar{u}\left(\tau_{2}\right), \gamma\left(\tau_{2}\right)\right)>0 .
\end{gathered}
$$


Because of $E V\left(\tau_{2}, \bar{u}\left(\tau_{2}\right), \gamma\left(\tau_{2}\right)\right)=\kappa \lambda \delta>\kappa \delta$ and $E V\left(t_{m}, \bar{u}\left(t_{m}\right), \gamma\left(t_{m}\right)\right) \leq \kappa \varepsilon$, there is $\tau_{3} \in\left[t_{m}, \tau_{2}\right)$ such that

$$
\begin{gathered}
E V\left(\tau_{3}, \bar{u}\left(\tau_{3}\right), \gamma\left(\tau_{3}\right)\right)=\kappa \delta, \\
E V(t, \bar{u}(t), \gamma(t)) \leq \kappa \delta, \quad t \in\left[\tau_{3}, \tau_{2}\right] . \\
D^{+} E V\left(\tau_{3}, \bar{u}\left(\tau_{3}\right), \gamma\left(\tau_{3}\right)\right)>0 .
\end{gathered}
$$

Noticing $D^{+} E V(t, \bar{u}(t), \gamma(t)) \leq \beta E V(t, \bar{u}(t), \gamma(t))$, we obtain

$$
\frac{D^{+} E V(t, \bar{u}(t), \gamma(t))}{E V(t, \bar{u}(t), \gamma(t))} \leq \beta
$$

Integrating both sides of (3.28) on $t \in\left[\tau_{3}, \tau_{2}\right]$, we claim that

$$
\int_{\tau_{3}}^{\tau_{2}} \frac{D^{+} E V(s, \bar{u}(s), \gamma(s))}{E V(s, \bar{u}(s), \gamma(s))} d s \leq \int_{\tau_{3}}^{\tau_{2}} \beta d s<\beta\left(t_{m+1}-t_{m}\right)<\ln \lambda
$$

However,

$$
\int_{\tau_{3}}^{\tau_{2}} \frac{D^{+} E V(s, \bar{u}(s), \gamma(s))}{E V(s, \bar{u}(s), \gamma(s))} d s=\int_{E V\left(\tau_{3}, \bar{u}\left(\tau_{3}\right), \gamma\left(\tau_{3}\right)\right)}^{E V\left(\tau_{2}, \bar{u}\left(\tau_{2}\right), \gamma\left(\tau_{2}\right)\right)} \frac{d \eta}{\eta}=\int_{\kappa \delta}^{\kappa \lambda \delta} \frac{d \eta}{\eta}=\ln (\kappa \lambda \delta)-\ln (\kappa \delta)=\ln \lambda
$$

This leads to a contradiction. Then, we have

$$
E V(t, \bar{u}(t), \gamma(t)) \leq \kappa \curlywedge \delta, \quad t_{m} \leq t<t_{m+1}
$$

Moreover,

$$
\begin{aligned}
E V\left(t_{m+1}, \bar{u}\left(t_{m+1}\right), \gamma\left(t_{m+1}\right)\right) & \leq E \kappa \bar{u}\left(t_{m+1}\right)=\kappa E\left\|H_{m+1}\left(u\left(t_{m+1}^{-}, x\right)\right)\right\|_{G}^{2} \leq \kappa \Gamma_{m+1}^{2} E\left\|u\left(t_{m+1}^{-}, x\right)\right\|_{G}^{2} \\
& \leq \frac{\kappa \Gamma_{m+1}^{2}}{\alpha} E V\left(t_{m+1}, \bar{u}\left(t_{m+1}^{-}\right), \gamma\left(t_{m+1}\right)\right) \leq \frac{\kappa \lambda \delta}{\lambda_{m+1}} \leq \kappa \delta .
\end{aligned}
$$

Therefore,

$$
E V(t, \bar{u}(t), \gamma(t)) \leq \kappa \lambda \delta, \quad t \geq 0,
$$

which results in

$$
\alpha E \bar{u}(t) \leq E V(t, \bar{u}(t), \gamma(t)) \leq \kappa \lambda \delta, \quad t \geq 0
$$


Namely,

$$
E\left\{\|u(t, x ; 0, \varphi)\|_{G}^{2}\right\}<\varepsilon, \quad t \geq 0
$$

This ends the proof of Theorem 3.1.

As an application, we consider a class of semilinear impulsive stochastic reactiondiffusion equations with Markovian switching as follows:

$$
\begin{aligned}
d u(t, x)= & {[C(\gamma(t)) \Delta u(t, x)+A(\gamma(t)) u(t, x)] d t } \\
& +\sigma(t, x, u, \gamma(t)) d w(t), \quad t \geq 0, t \neq t_{k}, x \in G, \\
u\left(t_{k}, x\right)= & H_{k}\left(u\left(t_{k}^{-}, x\right), \gamma\left(t_{k}\right)\right), \quad x \in G,
\end{aligned}
$$

with boundary condition

$$
\left.\frac{\partial u}{\partial N}\right|_{\partial G}=0, \quad t \geq 0
$$

and initial condition

$$
u\left(0, x, i_{0}\right)=\varphi(x), \quad x \in G, i_{0} \in \mathbb{S}
$$

where $C(\gamma(t))=\operatorname{diag}\left\{c_{1}(\gamma(t)), \ldots, c_{n}(\gamma(t))\right\}=\operatorname{diag}\left\{c_{i 1}, \ldots, c_{i n}\right\}$ with $c_{i j} \geq 0$ for $i \in \mathbb{S}, j=$ $1,2, \ldots, n . A(\gamma(t))=A_{i}=\left(a_{l j}(\gamma(t))\right)_{n \times n}=\left(a_{l j}^{(i)}\right)_{n \times n}, i \in \mathbb{S}$, is matrices. The remainder of system (3.36)-(3.39) is the same as that defined in system (2.1)-(2.4).

Theorem 3.2. Assume that

(A5) $\lambda>1$, where $\lambda=\inf \left\{\lambda_{k} \mid \lambda_{k}=1 / \Gamma_{k}^{2}, k=1,2, \ldots\right\}$,

(A6) $2 \max _{i}\left\{\lambda_{\max }\left(A_{i}\right)\right\}\left(t_{k}-t_{k-1}\right)<\ln \lambda, k=1,2 \ldots, i \in \mathbb{S}$.

Then, the trivial solution $u=0$ of system (3.36)-(3.39) is stable in mean square.

Proof. Construct a Lyapunov function $V(t, \bar{u}(t), \gamma(t))=\bar{u}(t)$, and compute the operator $\mathcal{L V}(t, \bar{u}(t), \gamma(t))$ that

$$
\mathcal{L V}(t, \bar{u}(t), \gamma(t))=\int_{G} 2 u^{\top} C(\gamma(t)) \Delta u d x+\int_{G} 2 u^{\top} A(\gamma(t)) u(t, x) d x
$$


By Green formula, we get

$$
\begin{aligned}
\int_{G} 2 u^{\top} C_{i} \Delta u d x & =2 \int_{G} \sum_{j=1}^{n} u_{j} c_{i j} \Delta u_{j} d x=2 \sum_{j=1}^{n} \int_{G} u_{j} c_{i j} \Delta u_{j} d x \\
& =2 \sum_{j=1}^{n} \int_{\partial \Omega} u_{j} c_{i j} \frac{\partial u_{j}}{\partial N} d s-2 \sum_{j=1}^{n} \int_{G} c_{i j} \nabla u_{j} \cdot \nabla u_{j} d x
\end{aligned}
$$

It follows from boundary condition that

$$
\sum_{j=1}^{n} \int_{\partial \Omega} u_{j} c_{i j} \frac{\partial u_{j}}{\partial N} d s=0
$$

Thus,

$$
\int_{G} 2 u^{\top} C_{i} \Delta u d x=-2 \sum_{j=1}^{n} \int_{G} c_{i j} \nabla u_{j} \cdot \nabla u_{j} d x \leq 0
$$

Therefore,

$$
\mathrm{LV}(t, \bar{u}(t), \gamma(t)) \leq 2 \int_{G} u^{\top} A(\gamma(t)) u(t, x) d x \leq 2 \max _{i}\left\{\lambda_{\max }\left(A_{i}\right)\right\} \bar{u}(t) .
$$

According to Theorem 3.1, we find that the trivial solution of system (3.36)-(3.39) is stable in mean square.

\section{Example}

Consider the following two dimension Markovian jumping impulsive stochastic reaction diffusion systems with two modes. The parameters are given as follows: Let $|G|=1 / 8$, when $r(t)=1$, we have

$$
\begin{aligned}
\left(\begin{array}{l}
d u_{1}(t, x) \\
d u_{2}(t, x)
\end{array}\right)= & \left(\begin{array}{cc}
3.4 & 0 \\
0 & 5.5
\end{array}\right)\left(\begin{array}{l}
\Delta u_{1}(t, x) \\
\Delta u_{2}(t, x)
\end{array}\right)+\left(\begin{array}{cc}
2.6 & 0 \\
0 & 1.7
\end{array}\right)\left(\begin{array}{l}
u_{1}(t, x) \\
u_{2}(t, x)
\end{array}\right) \\
& +\left(\begin{array}{c}
2 u_{1}(t, x) \\
-u_{2}(t, x)
\end{array}\right) d w(t), \quad t \geq 0, t \neq t_{k}, \\
\left(\begin{array}{l}
u_{1}\left(t_{k}, x\right) \\
u_{2}\left(t_{k}, x\right)
\end{array}\right)= & e^{-0.1 k}\left(\begin{array}{cc}
0.5 & -0.2 \\
0.3 & 0.6
\end{array}\right)\left(\begin{array}{l}
u_{1}\left(t_{k}^{-}, x\right) \\
u_{2}\left(t_{k^{\prime}}^{-} x\right)
\end{array}\right),
\end{aligned}
$$


when $r(t)=2$

$$
\begin{aligned}
\left(\begin{array}{l}
d u_{1}(t, x) \\
d u_{2}(t, x)
\end{array}\right)= & \left(\begin{array}{cc}
2.4 & 0 \\
0 & 2.5
\end{array}\right)\left(\begin{array}{l}
\Delta u_{1}(t, x) \\
\Delta u_{2}(t, x)
\end{array}\right)+\left(\begin{array}{cc}
1.6 & 0 \\
0 & 2.7
\end{array}\right)\left(\begin{array}{l}
u_{1}(t, x) \\
u_{2}(t, x)
\end{array}\right) \\
& +\left(\begin{array}{c}
2 u_{1}(t, x) \\
-u_{2}(t, x)
\end{array}\right) d w(t), \quad t \geq 0, t \neq t_{k}, \\
\left(\begin{array}{l}
u_{1}\left(t_{k}, x\right) \\
u_{2}\left(t_{k}, x\right)
\end{array}\right)= & e^{-0.1 k}\left(\begin{array}{cc}
0.6 & -0.3 \\
0.3 & 0.4
\end{array}\right)\left(\begin{array}{l}
u_{1}\left(t_{k}^{-}, x\right) \\
u_{2}\left(t_{k}^{-}, x\right)
\end{array}\right),
\end{aligned}
$$

where $t_{0}=0, t_{k}=t_{k-1}+0.1,(k=1,2, \ldots)$. By simple calculation, we obtain $\Gamma_{k}=0.6 e^{-0.1 k}, \lambda=$ $2.6>1,2 \lambda_{\max }\left(A_{i}\right)\left(t_{k}-t_{k-1}\right)<\ln \lambda=0.856$. From Theorem 3.2, the trivial solution of this system is stable in mean square.

\section{Conclusion}

In this paper, we discuss mean square stability of stochastic reaction diffusion equations with Markovian switching and impulsive perturbations, by means of Lyapunov function and stochastic analysis. As an application, we investigate a class of semilinear impulsive stochastic reaction-diffusion equations with Markovian switching and establish the stability criterion. Finally, we provide an example to demonstrate the effectiveness and efficiency of the obtained results.

\section{Acknowledgments}

The authors would like to thank the editors and the anonymous reviewers for their valuable comments and constructive suggestions. This research is supported by the National Natural Science Foundations of China (60974025, 60939003), National 863 Plan Project (2008AA04Z401, 2009AA043404), the Natural Science Foundation of Shandong Province (no. Y 2007G30), the Natural Science Foundation of Guangxi Autonomous Region (no. 2012GXNSFBA053003), the Scientific and Technological Project of Shandong Province (no. 2007GG3WZ04016), the Natural Scientific Research Innovation Foundation in Harbin Institute of Technology (HIT.NSRIF.2001120), the China Postdoctoral Science Foundation (20100481000) and the Shandong Provincial Key Laboratory of Industrial Control Technique (Qingdao University).

\section{References}

[1] N. N. Krasovskiı̌ and È. A. Lidskiı̌, "Analytical design of controllers in systems with random attributes. I. Statement of the problem, method of solving," Automation and Remote Control, vol. 22, pp. 1021-1025, 1961.

[2] P. Balasubramaniam, A. Manivannan, and R. Rakkiyappan, "Exponential stability results for uncertain neutral systems with interval time-varying delays and Markovian jumping parameters," Applied Mathematics and Computation, vol. 216, no. 11, pp. 3396-3407, 2010.

[3] P. Shi and E. K. Boukas, " $H_{\infty}$-control for Markovian jumping linear systems with parametric uncertainty," Journal of Optimization Theory and Applications, vol. 95, no. 1, pp. 75-99, 1997. 
[4] L. Zhang and E.-K. Boukas, "Mode-dependent $H_{\infty}$ filtering for discrete-time Markovian jump linear systems with partly unknown transition probabilities," Automatica, vol. 45, no. 6, pp. 1462-1467, 2009.

[5] L. Zhang and J. Lam, "Necessary and sufficient conditions for analysis and synthesis of Markov jump linear systems with incomplete transition descriptions," IEEE Transactions on Automatic Control, vol. 55, no. 7, pp. 1695-1701, 2010.

[6] L. Zhang, E.-K. Boukas, and J. Lam, "Analysis and synthesis of Markov jump linear systems with time-varying delays and partially known transition probabilities," IEEE Transactions on Automatic Control, vol. 53, no. 10, pp. 2458-2464, 2008.

[7] X. Mao and C. Yuan, Stochastic Differential Equations with Markovian Switching, Imperial College Press, London, UK, 2006.

[8] X. Mao, Y. Shen, and C. Yuan, "Almost surely asymptotic stability of neutral stochastic differential delay equations with Markovian switching," Stochastic Processes and their Applications, vol. 118, no. 8, pp. 1385-1406, 2008.

[9] X. Mao, A. Matasov, and A. B. Piunovskiy, "Stochastic differential delay equations with Markovian switching," Bernoulli, vol. 6, no. 1, pp. 73-90, 2000.

[10] X. Mao and L. Shaikhet, "Delay-dependent stability criteria for stochastic differential delay equations with Markovian switching," Stability and Control, vol. 3, no. 2, pp. 88-102, 2000.

[11] X. Mao, "Exponential stability of stochastic delay interval systems with Markovian switching," IEEE Transactions on Automatic Control, vol. 47, no. 10, pp. 1604-1612, 2002.

[12] X. Mao, "Stochastic functional differential equations with Markovian switching," Functional Differential Equations, vol. 6, no. 3-4, pp. 375-396, 1999.

[13] L. Huang and X. Mao, "On input-to-state stability of stochastic retarded systems with Markovian switching," IEEE Transactions on Automatic Control, vol. 54, no. 8, pp. 1898-1902, 2009.

[14] S. Wu, D. Han, and X. Meng, " $p$-moment stability of stochastic differential equations with jumps," Applied Mathematics and Computation, vol. 152, no. 2, pp. 505-519, 2004.

[15] H. Wu and J. Sun, " $p$-moment stability of stochastic differential equations with impulsive jump and Markovian switching," Automatica, vol. 42, no. 10, pp. 1753-1759, 2006.

[16] V. Lalshmikantham, D. Bainov, and P. Simeonov, Theory of Impulsive Diffusin Equation, World Scientific, Singapore, 1989.

[17] Q. Wang and X. Liu, "Exponential stability for impulsive delay differential equations by Razumikhin method," Journal of Mathematical Analysis and Applications, vol. 309, no. 2, pp. 462-473, 2005.

[18] Y. Zhang and J. Sun, "Stability of impulsive infinite delay differential equations," Applied Mathematics Letters, vol. 19, no. 10, pp. 1100-1106, 2006.

[19] D. Xu and Z. Yang, "Impulsive delay differential inequality and stability of neural networks," Journal of Mathematical Analysis and Applications, vol. 305, no. 1, pp. 107-120, 2005.

[20] L. Xu and D. Xu, "Mean square exponential stability of impulsive control stochastic systems with time-varying delay," Physics Letters A, vol. 373, no. 3, pp. 328-333, 2009.

[21] Z. Yang, D. Xu, and L. Xiang, "Exponential p-stability of impulsive stochastic differential equations with delays," Physics Letters A, vol. 359, no. 2, pp. 129-137, 2006.

[22] J. Yang, S. Zhong, and W. Luo, "Mean square stability analysis of impulsive stochastic differential equations with delays," Journal of Computational and Applied Mathematics, vol. 216, no. 2, pp. 474-483, 2008.

[23] B. Li, "The attracting set for impulsive stochastic difference equations with continuous time," Applied Mathematics Letters, vol. 25, no. 8, pp. 1166-1171, 2012.

[24] L. Pan and J. Cao, "Exponential stability of impulsive stochastic functional differential equations," Journal of Mathematical Analysis and Applications, vol. 382, no. 2, pp. 672-685, 2011.

[25] S. Zhang, J. Sun, and Y. Zhang, "Stability of impulsive stochastic differential equations in terms of two measures via perturbing Lyapunov functions," Applied Mathematics and Computation, vol. 218, no. 9, pp. 5181-5186, 2012.

[26] P. Cheng and F. Deng, "Global exponential stability of impulsive stochastic functional differential systems," Statistics E Probability Letters, vol. 80, no. 23-24, pp. 1854-1862, 2010.

[27] C. Li, J. Shi, and J. Sun, "Stability of impulsive stochastic differential delay systems and its application to impulsive stochastic neural networks," Nonlinear Analysis A, vol. 74, no. 10, pp. 3099-3111, 2011.

[28] H. Zhang, M. Dong, Y. Wang, and N. Sun, "Stochastic stability analysis of neutral-type impulsive neural networks with mixed time-varying delays and Markovian jumping," Neurocomputing, vol. 73, no. 13-15, pp. 2689-2695, 2010. 
[29] H. Bao and J. Cao, "Stochastic global exponential stability for neutral-type impulsive neural networks with mixed time-delays and Markovian jumping parameters," Communications in Nonlinear Science and Numerical Simulation, vol. 16, no. 9, pp. 3786-3791, 2011.

[30] Q. Zhu and J. Cao, "Stability of Markovian jump neural networks with impulse control and time varying delays," Nonlinear Analysis, vol. 13, no. 5, pp. 2259-2270, 2012.

[31] P. Balasubramaniam, R. Krishnasamy, and R. Rakkiyappan, "Delay-dependent stability criterion for a class of non-linear singular Markovian jump systems with mode-dependent interval time-varying delays," Communications in Nonlinear Science and Numerical Simulation, vol. 17, no. 9, pp. 3612-3627, 2012.

[32] J. Liang and J. Gao, "Global expoential stability of reaction-diffusion recurrent neural networks with time-varying delays," Physics Letters A, vol. 314, pp. 434-442, 2003.

[33] Y. G. Kao, C. C. Gao, and W. Han, “Global exponential robust stability of reaction-diffusion interval neural networks with continuously distributed delays," Neural Computing and Applications, vol. 19, pp. 867-873, 2010.

[34] C. Wang, Y. Kao, and G. Yang, "Exponential stability of impulsive stochastic fuzzy reaction-diffusion Cohen-Grossberg neural networks with mixed delays," Neurocomputing, vol. 89, no. 15, pp. 55-63, 2012.

[35] Y. G. Kao, J. F. Guo, C. H. Wang, and X. Q. Sun, "Delay-dependent robust exponential stability of Marko-vian jumping reaction-diffusion Cohen-Grossberg neural networks with mixed delays," Journal of The Franklin Institute, vol. 349, no. 6, pp. 1972-1988, 2012.

[36] D. Li, D. He, and D. Xu, "Mean square exponential stability of impulsive stochastic reaction-diffusion Cohen-Grossberg neural networks with delays," Mathematics and Computers in Simulation, vol. 82, pp. 1531-1543, 2012.

[37] Q. Luo, F. Deng, J. Bao, B. Zhao, and Y. Fu, "Stabilization of stochastic Hopfield neural network with distributed parameters," Science in China F, vol. 47, no. 6, pp. 752-762, 2004.

[38] Q. Luo and Y. Zhang, "Almost sure exponential stability of stochastic reaction diffusion systems," Nonlinear Analysis A, vol. 71, no. 12, pp. e487-e493, 2009.

[39] Y.-T. Zhang, Q. Luo, and X.-H. Lai, "Stability in mean of partial variables for stochastic reaction diffusion systems," Nonlinear Analysis A, vol. 71, no. 12, pp. e550-e559, 2009.

[40] L. Wang, Z. Zhang, and Y. Wang, "Stochastic exponential stability of the delayed reaction-diffusion recurrent neural networks with Markovian jumping parameters," Physics Letters A, vol. 372, no. 18, pp. 3201-3209, 2008.

[41] C. Vidhya and P. Balasubramaniam, "On the stability of the stochastic parabolic Itô equation with delay and Markovian jump," Computers \& Mathematics with Applications, vol. 60, no. 7, pp. 1959-1963, 2010.

[42] P. Balasubramaniam and C. Vidhya, "Exponential stability of stochastic reaction-diffusion uncertain fuzzy neural networks with mixed delays and Markovian jumping parameters," Expert Systems with Applications, vol. 39, no. 3, pp. 3109-3115, 2012. 


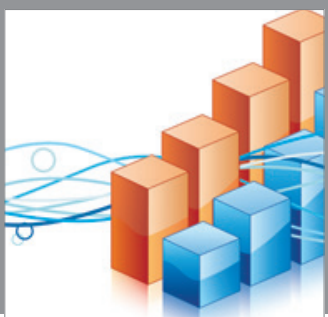

Advances in

Operations Research

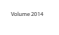

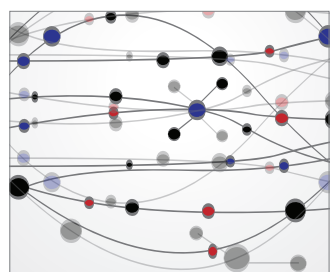

\section{The Scientific} World Journal
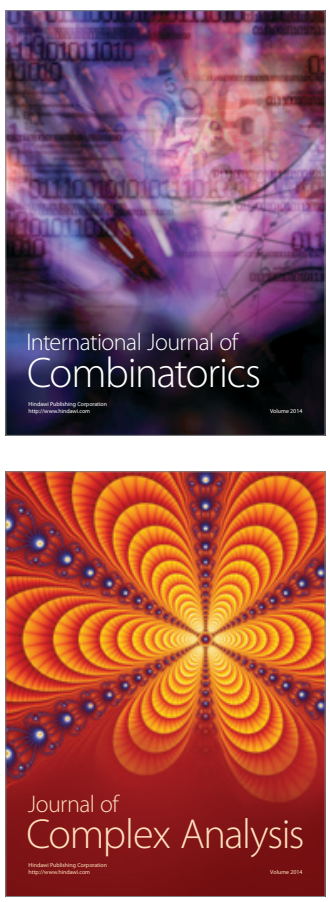

International Journal of

Mathematics and

Mathematical

Sciences
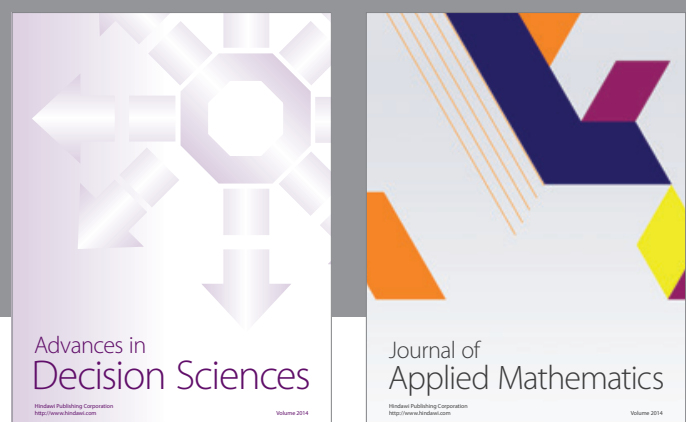

Journal of

Applied Mathematics
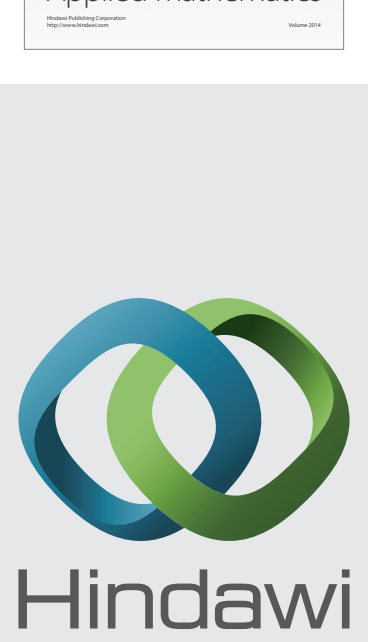

Submit your manuscripts at http://www.hindawi.com
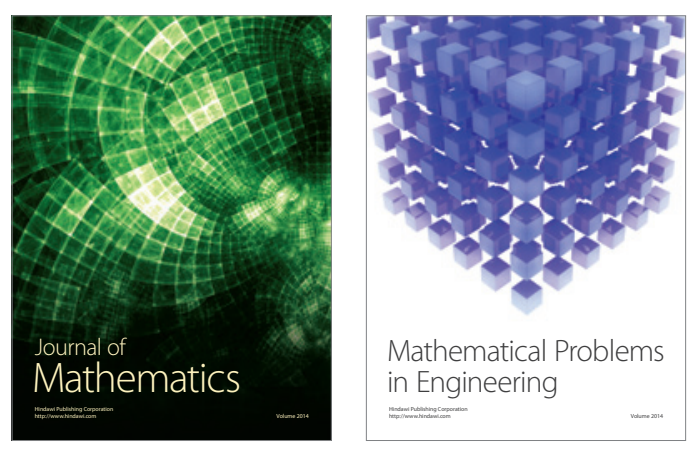

Mathematical Problems in Engineering
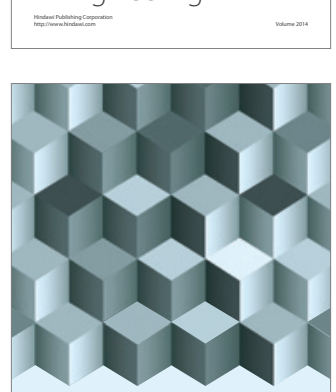

Journal of

Function Spaces
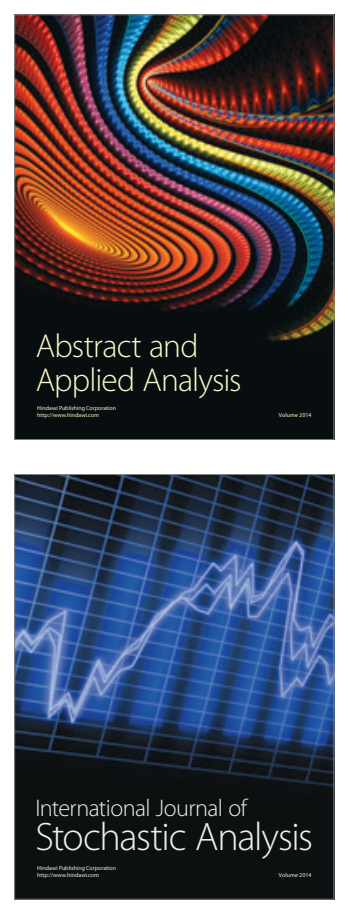

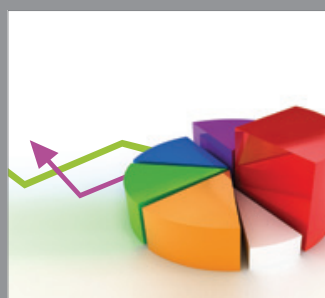

ournal of

Probability and Statistics

Promensencen
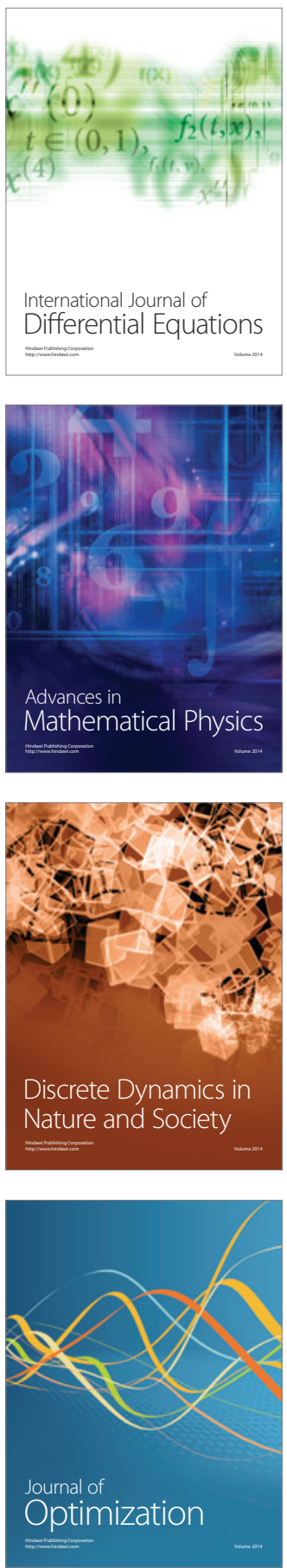